\title{
microRNA-22 acts as a metastasis suppressor by targeting metadherin in gastric cancer
}

\author{
YUNYUN TANG ${ }^{1,2^{*}}$, XIAOPING LIU ${ }^{3 *}$, BO SU ${ }^{1,2}$, ZHIWEI ZHANG ${ }^{1,2}$, XI ZENG $^{1,2}$, \\ YANPING LEI ${ }^{1,2}$, JIAN SHAN ${ }^{1,2}$, YONGJUN WU ${ }^{2,4}$, HAILIN TANG $^{3}$ and QI SU ${ }^{1,2}$ \\ ${ }^{1}$ Center for Gastric Cancer Research of Hunan Province, First Affiliated Hospital, University of South China; \\ ${ }^{2}$ Cancer Research Institute, University of South China, Key Laboratory of Cancer Cellular and Molecular Pathology of \\ Hunan Provincial University, Hengyang, Hunan 421001; ${ }^{3}$ Sun Yat-Sen University Cancer Center, State Key Laboratory \\ of Oncology in South China, Collaborative Innovation Center for Cancer Medicine, Guangzhou, Guangdong 510060; \\ ${ }^{4}$ Affiliated Xiangtan Hospital, University of South China, Xiangtan, Hunan 411100, P.R. China
}

Received December 23, 2013; Accepted August 29, 2014

DOI: $10.3892 / \mathrm{mmr} .2014 .2682$

\begin{abstract}
RNA (miR)-22 has been reported to be downregulated in hepatocellular, lung, colorectal, ovarian and breast cancer, acting as a tumor suppressor. The present study investigated the potential effects of miR-22 on gastric cancer invasion and metastasis and the molecular mechanism. miR-22 expression was examined in tumor tissues of in 89 gastric cancer patients by in situ hybridization (ISH) analysis. Additionally, the association between miR-22 levels and clinicopathological parameters was analyzed. A luciferase assay was conducted for target identification. The ability of invasion and metastasis of gastric cancer cells in vitro and in vivo was evaluated by cell migration and invasion assays and in a xenograft model. The results showed that miR-22 was downregulated in the gastric cancer specimens and significantly correlated with the advanced clinical stage and lymph node metastasis. In addition, metadherin (MTDH) was shown to be a direct target of miR-22 and the expression of MTDH was inversely correlated with miR-22 expression in gastric cancer. Ectopic expression of miR-22 suppressed cell invasion and metastasis in vitro and in vivo. The present study suggested that miR-22 may be
\end{abstract}

Correspondence to: Professor Qi Su, Center for Gastric Cancer Research of Hunan Province, Cancer Research Institute, University of South China, 28 West Changsheng Road, Hengyang, Hunan 421001, P.R. China

E-mail: suqi1945@163.com

Professor Hailin Tang, Sun Yat-Sen University Cancer Center, State Key Laboratory of Oncology in South China, Collaborative Innovation Center for Cancer Medicine, 651 East Dongfeng Road, Guangzhou, Guangdong 510060, P.R. China

E-mail: tanghl@sysucc.org.cn

*Contributed equally

Key words: microRNA-22, gastric cancer, metadherin, metastasis a valuable prognostic factor in gastric cancer. miR-22 inhibited gastric cancer cell invasion and metastasis by directly targeting MTDH. The novel miR-22/MTDH link confirmed in the present study provided a novel, potential therapeutic target for the treatment of gastric cancer.

\section{Introduction}

Gastric cancer is the fourth most prevalent type of cancer worldwide (1). Radical gastrectomy is the only effective treatment for gastric cancer at an early stage. However, in most cases, gastric cancer is at an advanced stage at the time of diagnosis, and the therefore missed opportunity for radical operation results in high mortality (2). Thus, the discovery of effective biomarkers and potential therapeutic targets have become highlighting fields in gastric cancer.

In recent years, microRNA (miRNA/miR) has been the focus of molecular biological studies. microRNAs are small nonprotein-coding RNAs of 22 nucleotides in length, which have critical roles in regulating expression of target genes post-transcription (3). miRNAs have been reported to be significantly involved in tumorigenesis and progression, acting as either oncogenes or tumor suppressors $(4,5)$. Emerging studies have reported that a number of miRNAs is commonly dysregulated in gastric cancer. For example, miR-429, miR-148a, miR-20b, miR-195, miR-378, miR-101 and miR-200b/c (5-9) were reported to always be down-regulated, while miR-19a, miR-301a, miR-544, miR-18a, miR-125b and miR-181b (10-15) were overexpressed in cancers. Since these specific miRNAs regulate different target genes which are involved in various signaling pathways and biological processes, gastric cancer is a complex disease with multiple dysregulated genes.

A previous study by our group used a miRNA microarray to screen for the differential miRNAs by treatment with diallyl disulfide in human gastric cancer cell lines and found that miR-22 was significantly increased following treatment (16). In addition, the same study by our group reported that overexpression of miR-22 inhibited gastric cancer cell growth in vitro and in vivo as well as induced apoptosis through targeting 
the Wnt-1 signaling pathway (16). Moreover, upregulation of miR-22 expression has been reported to suppress gastric cancer cell migration and invasion by targeting the Sp1 gene (17).

The purpose of the present study was to investigate the prognostic role of miR-22 in gastric cancer patients and explore the potential molecular mechanism. Furthermore, it was shown that metadherin (MTDH) was a target gene for miR-22 and regulated the invasion and metastasis of gastric cancer cells. To the best of our knowledge, the present study was the first to examine the miR-22/ MTDH link in gastric cancer invasion and metastasis. These results provide profound understanding of the antitumor mechanism of miR-22.

\section{Materials and methods}

Cell culture. The gastric cancer cell line SGC-7901 was purchased from the Chinese Academy Medical Science (Beijing, China). These cells were grown in RPMI-1640 medium supplemented with $10 \%$ fetal bovine serum, penicillin and streptomycin (Gibco-BRL, NY, USA) and were cultured in a humidified chamber with $5 \% \mathrm{CO}_{2}$ at $37^{\circ} \mathrm{C}$. All transfections were performed using Lipofectamine 2000 (Invitrogen Life Technologies, Carlsbad, CA, USA).

Clinical samples. All tissue samples used in the present study were collected from the Hunan Provincial Tumor Hospital (Changsha, China). Written informed consent was acquired from all study participants. This study was approved by the Ethics Committee of University of South China Cancer Center Health Authority (Hengyang, China). The collection and use of tissues was performed following procedures in accordance with the ethical standards as formulated in the Helsinki Declaration.

The tissue microarrays (TMA), including 89 cases of gastric cancer and 41 cases of normal stomach mucosa, were used for in situ hybridization (ISH) analysis. All data, including age, gender, histological grade, tumor size, invasion depth (T stage) and lymph node metastasis, were obtained from clinical and pathological records.

ISH analysis. miR-22 miRCURY LNA ${ }^{\mathrm{TM}}$ detection probe (Exiqon, Vedbaek, Denmark) was used for ISH according to a standard method, as described previously (5). The 5'-3' sequence (enhanced with locked nucleic acid) was ACAGTTCTTCAACTGGCAGCTT with a digoxigenin label at both the $5^{\prime}$ and $3^{\prime}$ ends.

MTDH silencing and enhancement of MTDH expression using a vector. The sense sequence of small interfering (si)RNA oligonucleotides targeting the MTDH transcripts was as follows: si-MTDH sense, 5'-ACGGUACCCCGGCUGGGUGUU-3' and antisense, 5'-UUUGCCAUGGGGCCGACCCAC-3' (Invitrogen Life Technologies). Control siRNA was used as a negative control. Cells were plated in culture dishes for $24 \mathrm{~h}$ and transfected with siRNA using Lipofectamine 2000 following the manufacturer's instructions. Following $48 \mathrm{~h}$, the cells were harvested for use in other assays or for RNA and protein extraction.

MTDH-expressing vector was constructed. Full-length MTDH cDNA [OmicsLink ${ }^{\mathrm{TM}}$ Expression Clone (CMV promoter), pReceiver-M02] was purchased from GeneCopoeia $^{\mathrm{TM}}$ (Rockville, MD, USA) and was subcloned into the eukaryotic expression vector pcDNA3.1(+) (GeneCopoeia, Rockville, MD, USA). The vector pcDNA3.1(+) was used as a negative control.

In vivo metastasis assays. To assay the effect of miR-22 on tumor metastasis, $1 \times 10^{6}$ SGC-7901 cells were infected with miR-22 or control viruses and injected into the tail vein of nude mice (eight in each group). The mice were maintained under a controlled temperature $\left(21^{\circ} \mathrm{C}-23^{\circ} \mathrm{C}\right)$ and $12 \mathrm{~h} \mathrm{light} /$ dark cycle, and were housed in a laminar flow cabinet under pathogenfree conditions, in standard vinyl cages with air filter tops. Cages, bedding and water containers were autoclaved prior to use. After 45 days, necropsies were performed. Lung tissue samples were fixed with formalin and embedded in paraffin. Tissue slides were prepared from the tissue samples with a $4 \mu \mathrm{m}$ thickness. The numbers of micrometastases in hematoxylin and eosin-stained lung tissue sections in individual mice were analyzed by morphological observation. Briefly, following deparaffinization and dehydration, the slides were soaked in a solution of $90 \%$ methanol $/ 3 \% \mathrm{H}_{2} \mathrm{O}_{2}$ for $15 \mathrm{~min}$ at room temperature, in order to block endogenous peroxidases. The slides were then treated with $0.01 \mathrm{~mol} / 1$ EDTA solution $(\mathrm{pH} 8)$ and incubated at $96^{\circ} \mathrm{C}$ for $4 \mathrm{~min}$, in an autoclave, for antigen retrieval. The following markers were used: rabbit polyclonal antibody MTDH (1:1000; Sigma-Aldrich, St. Louis, MO, USA), rabbit polyclonal antibody SP1 and rabbit polyclonal antibody $\beta$-actin (1:100; Santa Cruz Biotechnology, Inc., Dallas, TX, USA). The slides were incubated with the antibodies overnight at $4^{\circ} \mathrm{C}$, and then incubated at room temperature with the goat anti-mouse IgG secondary antibody (1:1000; Boster Biological Technology Co., Pleasanton, CA, USA). The animal handling and all experimental procedures were performed following approval of the Animal Ethics Committee of the University of South China (Hengyang, China).

Cell proliferation, migration and invasion and luciferase assays, immunohistochemistry and protein/mRNA analysis. MTT assay and real time quantitative polymerase chain reaction (RT-qPCR) analysis were performed according to standard methods, as described previously (16). Cell migration wound healing assay, Matrigel ${ }^{\mathrm{TM}}$ (BD Biosciences, Franklin Lakes, NJ, USA) invasion assay, western blotting with MTDH, SP1, and $\beta$-actin antibodies (1:50; Santa Cruz Biotechnology, Inc.) immunohistochemical analysis and luciferase activity assay (Promega Corporation, Madison, WI, USA) were performed according to a standard method, as described previously (5).

Statistical analysis. Values are presented as the mean \pm standard deviation unless otherwise noted. The t-test and $\mathrm{x}^{2}$ test were used for statistical analysis, with $\mathrm{P}<0.05$ considered to indicate a statistically significant difference between values. Statistical analyses were performed using SPSS 16.0 software (International Business Machines, Armonk, NY, USA).

\section{Results}

miR-22 is downregulated in gastric cancer and associated with advanced clinical stage and lymph node metastasis. 
Table I. miR-22 is downregulated in primary gastric cancer.

miR-200b

\begin{tabular}{lcccr}
\cline { 3 - 4 } Viable & Cases (n) & Low (n) & High (n) & P-value \\
\hline Normal & 41 & 15 & 26 & 0.006 \\
Gastric cancer & 89 & 55 & 34 & \\
\hline
\end{tabular}

Table II. Analysis of the correlation between expression of miR-22 in primary gastric cancer and its clinicopathological parameters.

\begin{tabular}{|c|c|c|c|c|}
\hline \multirow[b]{2}{*}{ Viable } & \multirow[b]{2}{*}{ Cases (n) } & \multicolumn{3}{|c|}{ miR-200b } \\
\hline & & Low (n) & High (n) & P-value \\
\hline \multicolumn{5}{|l|}{ Age (years) } \\
\hline$<60$ & 54 & 31 & 23 & 0.202 \\
\hline$\geq 60$ & 35 & 24 & 11 & \\
\hline \multicolumn{5}{|l|}{ Gender } \\
\hline Male & 48 & 27 & 21 & 0.172 \\
\hline Female & 41 & 28 & 13 & \\
\hline \multicolumn{5}{|l|}{ Histological grade } \\
\hline Good and moderate & 21 & 13 & 8 & 0.601 \\
\hline Poor and other & 68 & 42 & 26 & \\
\hline \multicolumn{5}{|l|}{ T stage } \\
\hline $\mathrm{T} 1-\mathrm{T} 2$ & 49 & 30 & 19 & 0.539 \\
\hline T3-T4 & 40 & 25 & 15 & \\
\hline \multicolumn{5}{|l|}{ TNM stage } \\
\hline I-II & 33 & 16 & 17 & 0.040 \\
\hline III-IV & 56 & 39 & 17 & \\
\hline \multicolumn{5}{|l|}{ Lymph node metastasis } \\
\hline Present & 64 & 49 & 15 & $<0.001$ \\
\hline Absent & 25 & 6 & 19 & \\
\hline
\end{tabular}

miR-22 has been shown to be downregulated in various cancers, including gastric cancer $(16,18-20)$. Recently, Wang et al (21)found that miR-22 is associated with lymph node metastasis and advanced clinical stage, as well as distant metastasis in gastric cancer by RT-qPCR analysis (21). To further investigate the relevance between miR-22 expression and gastric cancer metastasis, miR-22 expression was assessed in 89 gastric cancer and 41 normal stomach tissues by ISH analysis using a tissue microarray (TMA). Among 41 normal stomach samples, 26 (63\%) exhibited high expression of miR-22. By contrast, 63\% (55 out of 89) of gastric cancer specimens exhibited low expression of miR-22 (Table I). Next, the correlation between clinicopathological parameters and altered miR-22 expression was assessed. Clinical samples were subsequently divided into low and high expression groups based on miR-22 expression scores $>2$ or $<2$, respectively. As shown in Table II, miR-22 levels were inversely correlated with the tumor stage $(\mathrm{P}=0.03)$ and lymph node metastasis $(\mathrm{P}=0.001)$.
miR-22 overexpression suppresses gastric cancer cell invasion and metastasis in vitro and vivo. To investigate the effect of miR-22 on the invasion potential of gastric cancer cells, ectopic miR-22 mimics were transfected into gastric cancer cells. RT-qPCR analysis demonstrated that the transfection was successful in SGC-7901 cells (data not shown). A Transwell assay was employed to assess the impact of miR-22 on cellular invasion in SGC-7901 cells. Ectopic expression of miR-22 significantly inhibited SGC-7901 cell invasion capacity compared with that of the control group (Fig. 1A and B). Next, the role of miR-22 in tumorigenesis was investigated by using nude mouse xenograft models. SGC-7901 cells infected with miR-22 or control lentivirus were injected into the tail veins of nude mice to examine the formation of lung metastasis. As shown Fig. 1C, a significantly lower number of macroscopic lung metastases was observed for miR-22-overexpressing cells than for control cells. These results indicated that miR-22 may repress gastric cancer invasion and metastasis. 
A

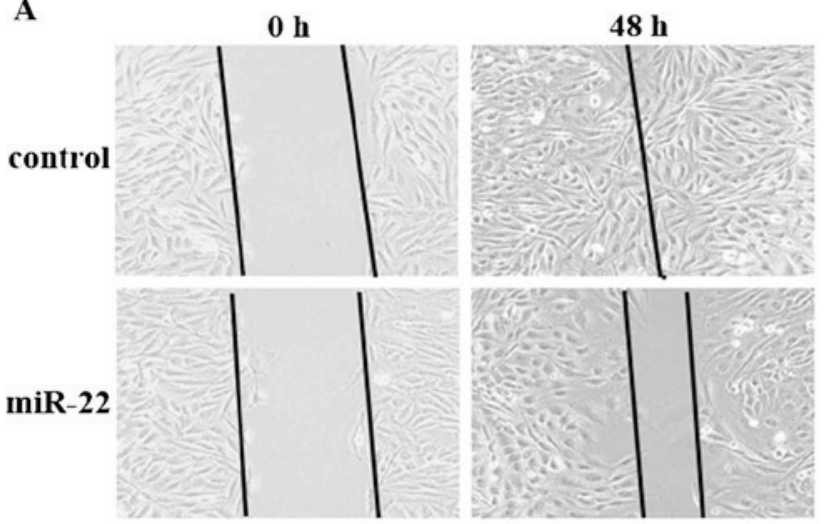

B

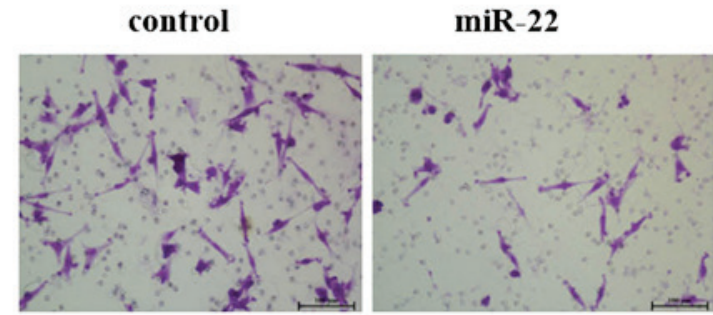

control

C

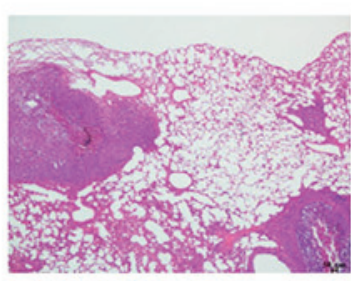

miR-22

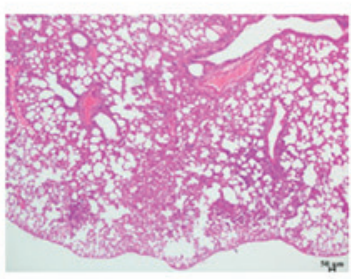

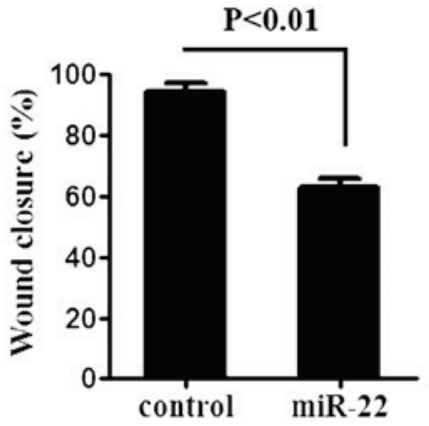
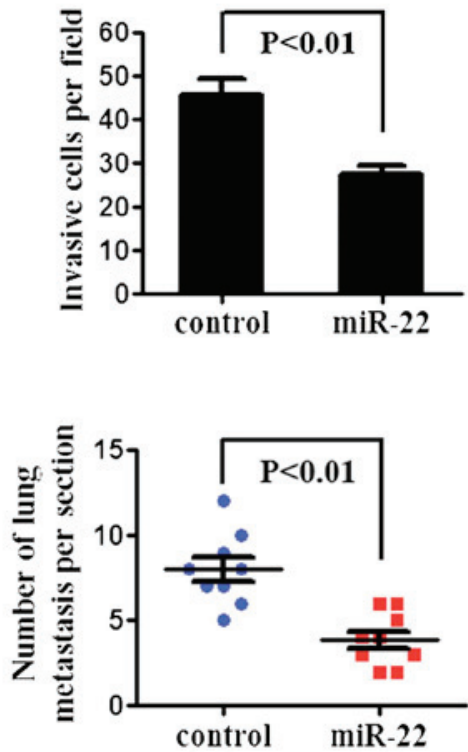

Figure 1. miR-22 suppresses gastric cancer cell invasion in vitro and metastasis in vivo. (A) Wound healing assay of SGC-7901 cells infected with miR-22 mimics or control. (B) Invasion assay of SGC-7901 cells infected with miR-22 mimics or control. Invasion was measured by means of Transwell assays with Matrigel. (C) Tumor metastasis in mouse xenograft models. SGC-7901 cells overexpressing miR-22 or control cells were injected into the tail veins of nude mice. After 45 days, micrometastases in hematoxylin and eosin-stained lung tissue sections from mice ( $\mathrm{n}=8 / \mathrm{group})$ were assessed (magnification, $\mathrm{x} 100)$. Values are expressed as the mean \pm standard error of the mean. miR, microRNA. Magnification, x100.

$M T D H$ is a direct target of $m i R-22$. To explore the molecular mechanism of miR-22 in gastric cancer, the TargetScan algorithm was used to identify putative protein-coding gene targets of miR-22, indicating that MTDH is a putative target of miR-22. Moreover, TargetScan analysis for MTDH revealed two possible binding sites for miR-22 (Fig. 2A). To ascertain whether MTDH was a direct target of miR-22, luciferase reporter assays were performed. The MTDH 3' untranslated (UTR) region was cloned into a luciferase reporter vector and the corresponding mutant counterparts of the two binding sites were directly fused to the downstream end of the firefly luciferase gene. Co-transfection of SGC-7901 cells with MTDH-3'UTR/pmirGLO and miR-22 mimics or a control were co-transfected into SGC-7901 cells. Luciferase activity was measured $48 \mathrm{~h}$ following transfection. Co-transfection with MTDH reporter and miR-22 resulted in $\sim 43 \%$ reduction of the luciferase activity with respect to cotransfection with the control (Fig. 2B). However, in cells transfected with the vector carrying two mutations, luciferase activity was not significantly changed. Of note, miR-22 was able to target each of the binding sites of the MTDH-3'UTR. When one of the two sites was mutated, luciferase activity was reduced compared with that with mutation at two sites (Fig. 2B). To further confirm that MTDH was a target gene for miR-22, miR-22 mimic or a control were transfected into SGC-7901 cell lines, and qRT-PCR and western blot analysis were performed to detect the expression of MTDH regulated by miR-22 in SGC-7901 cells. A marked reduction in the mRNA and protein levels of MTDH and SP1 (a known target of miR-22) was observed in miR-22-transfected SGC-7901 cells compared with the negative control (Fig. 2C and D). In conclusion, these results indicated that miR-22 downregulated MTDH expression by directly targeting its 3 'UTR.

Expression of MTDH is inversely correlated with miR-22 expression in gastric cancer. As miR-22 is downregulated in gastric cancer and directly targets MTDH, the present study next determined whether MTDH protein expression is negatively associated with miR-22 levels in the gastric cancer tissue samples. Immunohistochemical staining was applied 
A
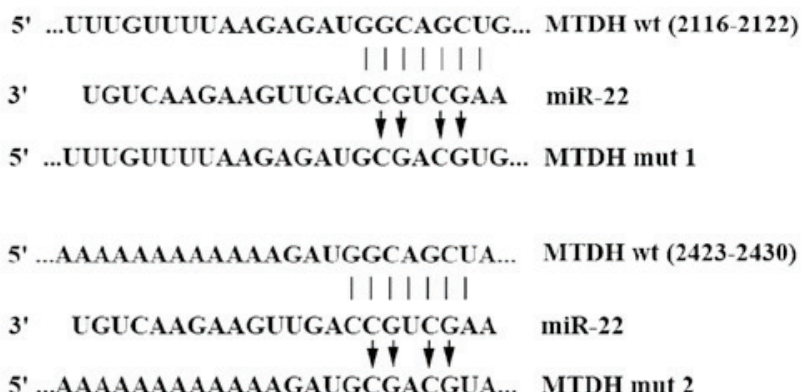

5 ...AAAAAAAAAAAAGAUGCGACGUA... MTDH mut 2
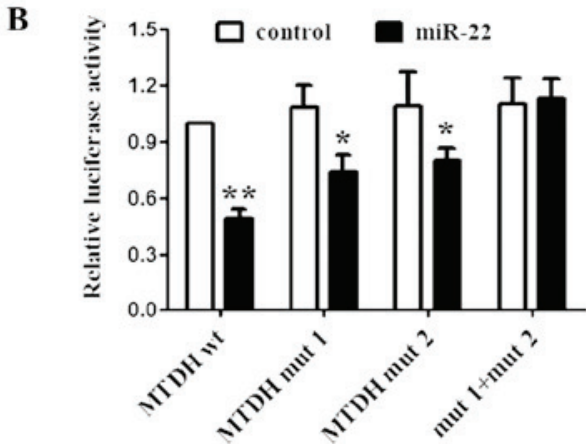

C
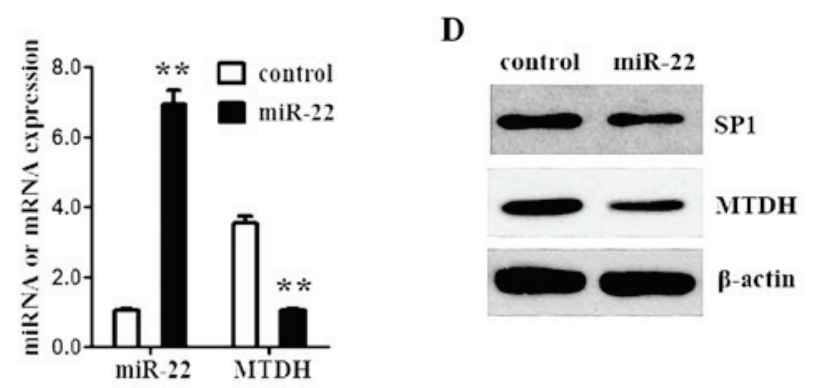

E

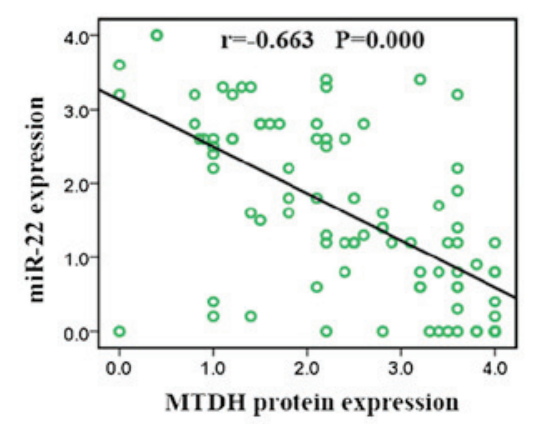

$\mathbf{F}$

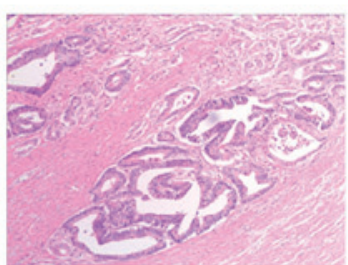

H E

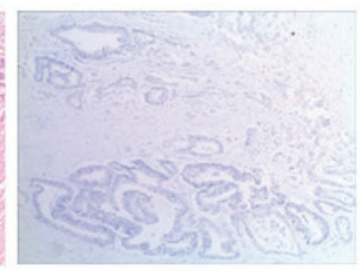

miR-22

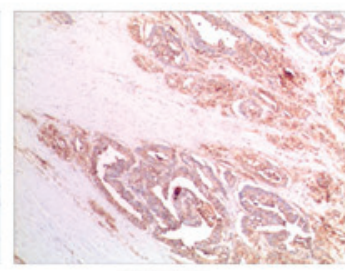

MTDH

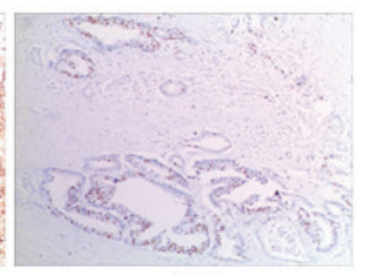

SP1

Figure 2. miR-22 directly targets MTDH. (A) Luciferase assay on SGC-7901 cells, which were cotransfected with miR-22 mimics and a luciferase reporter containing MTDH3'-UTR (MTDH-wt), MTDH3'-UTR (MTDH-mut1) and MTDH3'-UTR (MTDH -mut2) which carried a substitution of four nucleotides within the miR-22 binding site. An empty luciferase reporter construct was used as a negative control. ${ }^{\prime} \mathrm{P}<0.05$ vs. scramble. (B) The relative luciferase activity in SGC-7901 cells was determined following co-transfection of the MTDH3'-UTR or mutant plasmids with miR-22. (C and D) Effect of the miR-22 on the mRNA and protein expression of MTDH by polymerase chain reaction and western blot analyses. SP1 was used as a positive control. (E) Analysis of correlation of miR-22 and MTDH expression in gastric cancer tissues. (F) Detection of MTDH protein expression conducted on a tissue microarray by immunohistochemical staining. SP1 was used as a positive control. UTR, untranslated region; mut, mutated; wt, wild-type; MTDH, metadherin; miR, microRNA; HE, hematoxylin and eosin stain; SP1, specificity protein 1.Magnification, x100.

to detect the MTDH protein expression in gastric cancer and their corresponding nontumorous tissues. Correlation analysis showed that the levels of miR-200b were inversely correlated with the expression of MTDH in primary gastric cancer tissue (Fig. 2E). Furthermore, it was found that overexpression of MTDH was more frequent in gastric cancer with low levels of miR-22 (Fig. 2F), providing additional evidence for miR-22 mediated $\mathrm{MTDH}$ regulation.

miR-22 suppresses gastric cancer cell proliferation and invasion by targeting $M T D H$. A previous study by our group suggested that miR-22 suppresses gastric cancer cell proliferation in vitro and vivo (16) and the present study showed that miR-22 inhibits gastric cancer cell and invasion. To confirm the roles of MTDH as a critical mediator of miR-22 in gastric cancer cell metastasis, MTDH-siRNA or MTDH/pcDNA3.1 were used to knockdown or up-regulate MTDH expression in SGC-7901 cells, respectively. si-MTDH or MTDH/pcDNA3.1 significantly reduced or increased the expression of MTDH protein as confirmed by western blot (data not shown).
MTDH RNA interference with respect to transfection of the empty expression vector resulted in a marked decrease in the proliferation and invasive potential of SGC-7901 cells (Fig. 3A and B). However, the counterpart with transfection of MTDH/pcDNA3.1 displayed markedly increased cell proliferation and invasion in SGC-7901 cells. However, co-transfection with miR-22 mimics was able to suppress the capability of proliferation and invasion in SGC-7901 cells (Fig. 3A and B). These results suggested that miR-22 suppressed gastric cancer cell proliferation and invasion by targeting MTDH and that MTDH was a direct and functional target for miR-22.

\section{Discussion}

Recent studies have reported that miR-22 was frequently deregulated in various cancers. For instance, miR-22 was downregulated in gastric, hepatocellular, lung, colorectal and breast cancer as a tumor suppressor $(16,18-20)$, while in myelodysplastic syndrome (MDS) and leukemia, miR-22 was often upregulated, acting as an oncogene (22). In the present study, 
A

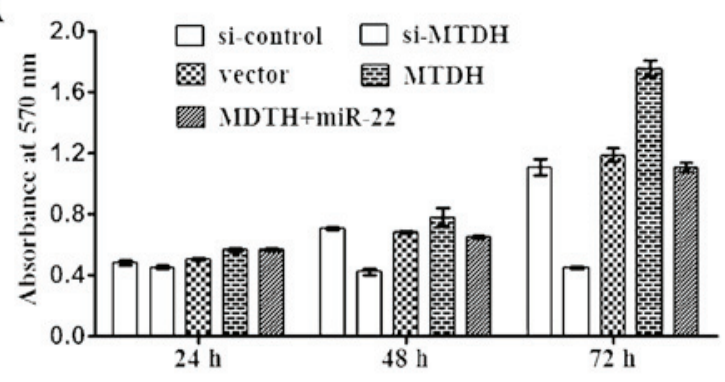

B

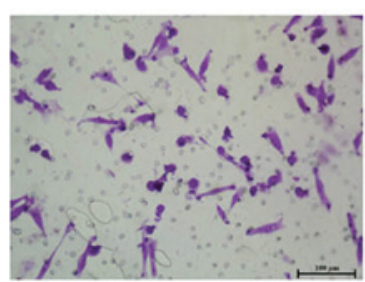

vector

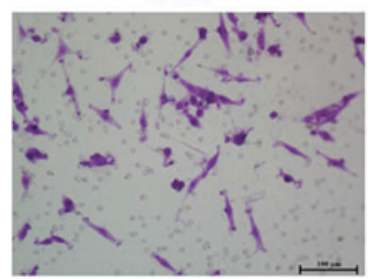

MTDH+miR-22

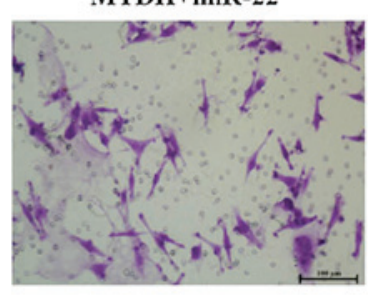

si-MTDH

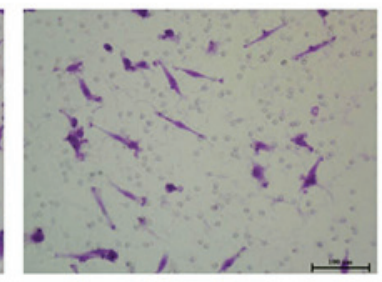

MTDH
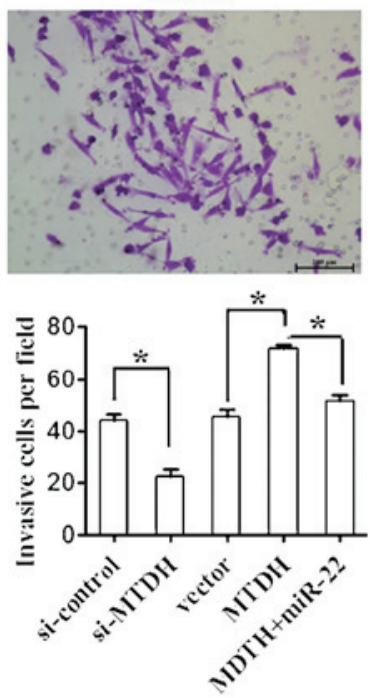

Figure 3. Knockdown or overexpression of MTDH reduces or increases invasion of gastric cancer cells, respectively. (A) Knockdown or overexpression of MTDH reduces or increases proliferation in gastric cancer cells and miR-22 can attenuate the proliferative potential of MTDH in SGC-7901, as demonstrated by MTT assay. (B) Knockdown or overexpression of MTDH reduces or increases invasion in gastric cancer cells and miR-22 and can attenuate the invasive potential of MTDH in SGC-7901. MTDH, metadherin; miR, microRNA; si, small interfering RNA.

the ISH assay further confirmed that miR-22 was frequently downregulated in gastric cancer tissues and was associated with advanced clinical stage and lymph node metastasis, which was consistent with a study by Wang et al (21). It was further shown that miR-22 inhibited gastric cancer cell invasion and metastasis in vitro and in vivo. These findings demonstrated that miR-22 served as a tumor suppressor and contributed to the development and metastasis in gastric cancer.

To the best of our knowledge, the present study provided the first evidence that miR-22 exerted its function by directly targeting MTDH. MTDH was initially identified as a human immunodeficiency virus-inducible gene in primary human fetal astrocytes (23) and was implicated in tumorigenesis and progression as an oncogene in numerous types of human

malignancies. A large number of studies show that MTDH is overexpressed in human cancers, including breast, , hepatocellular, prostate, bladder, cervical, endometrial and colorectal cancer as well as glioma (24-29). Furthermore, it has been reported that MTDH had important roles in multiple biological processes in the course of tumorigenesis via modulating of diverse signaling pathways, including the phosphoinositide 3 -kinase-AKT, nuclear factor- $\kappa$ light-chain-enhancer of activated $\mathrm{B}$ cells, mitogen-activated protein kinase, and Wnt/ $\beta$-catenin signaling pathways (30-32). More importantly, MTDH promotes metastasis through induction of the epithelial-mesenchymal transition in hepatocellular carcinoma and breast cancer $(33,34)$.

The present study found that MTDH was upregulated in gastric cancer and was negatively correlated with miR-22 levels in gastric cancer tissues. It was also shown that knockdown of MTDH in SGC-7901 cells was able to significantly decrease the cellular proliferation and invasion capability. Furthermore, the antitumor efficacy of miR-22 was evaluated in vivo by using a xenograft mouse model of gastric cancer. It was found that miR-22 not only inhibited gastric cancer growth, but also suppressed gastric cancer growth through regulating MTDH in vivo. Since deregulation of miR-22 in various cancers is frequently observed, miR-22 may have an important role in tumorigenesis and tumor progression. The present study found that gastric cancer tissue from patients with more advanced TNM stages and lymph node metastasis exhibited lower expression of miR-22, suggesting that low expression of miR-22 was associated with gastric cancer progression and metastasis.

In conclusion, the present study suggested that overexpression of the MTDH protein in gastric cancer may be the result of reduced levels of miR-22. Moreover, miR-22 can remarkably suppress gastric cancer cell proliferation and invasion. The herein verified miR-22/MTDH link provided a novel, potential therapeutic target for the treatment of gastric cancer.

\section{Acknowledgements}

The present study was supported by the Nature Scientific Foundation of China (nos. 31100935, 81102854, 81374013 and 81101643), The Key Hunan Provincial Natural Science Foundation of China (no. 07JJ3033) and The Construct Program of the Key Discipline in Hunan Province of China (no. 2011-76).

\section{References}

1. Jemal A, Bray F, Center MM, Ferlay J, Ward E and Forman D: Global cancer statistics. CA Cancer J Clin 61: 69-90, 2011.

2. Coburn NG: Lymph nodes and gastric cancer. J Surg Oncol 99: 199-206, 2009.

3. Tang H, Liu X, Wang Z, et al: Interaction of hsa-miR-381 and glioma suppressor LRRC4 is involved in glioma growth. Brain Res 1390: 21-32, 2011.

4. Cheng CJ and Slack FJ: The duality of oncomiR addiction in the maintenance and treatment of cancer. Cancer J 18: 232-237, 2012.

5. Tang H, Deng M, Tang Y, et al: miR-200b and miR-200c as prognostic factors and mediators of gastric cancer cell progression. Clin Cancer Res 19: 5602-5612, 2013.

6. Zheng B, Liang L, Wang C, et al: MicroRNA-148a suppresses tumor cell invasion and metastasis by downregulating ROCK1 in gastric cancer. Clin Cancer Res 17: 7574-7583,2011.

7. Liu D, Xia P, Diao D, et al: MiRNA-429 suppresses the growth of gastric cancer cells in vitro. J Biomed Res 26: 389-393, 2012. 
8. He XP, Shao Y, Li XL, et al: Downregulation of miR-101 in gastric cancer correlates with cyclooxygenase- 2 overexpression and tumor growth. FEBS J 279: 4201-4212, 2012.

9. Zhang L, Liu X, Jin H, et al: miR-206 inhibits gastric cancer proliferation in part by repressing cyclinD2. Cancer Lett 332: 94-101, 2013.

10. Qin S, Ai F, Ji WF, Rao W, Zhang HC and Yao WJ: miR-19a promotes cell growth and tumorigenesis through targeting SOCS1 in gastric cancer. Asian Pac J Cancer Prev 14: 835-840, 2013.

11. Wang M, Li C, Yu B, et al: Overexpressed miR-301a promotes cell proliferation and invasion by targeting RUNX3 in gastric cancer. J Gastroenterol 48: 1023-1033, 2013.

12. Wu W, Takanashi M, Borjigin N, et al: MicroRNA-18a modulates STAT3 activity through negative regulation of PIAS3 during gastric adenocarcinogenesis. Br J Cancer 108: 653-661, 2013.

13. Yang ZX, Lu CY, Yang YL, Dou KF and Tao KS: MicroRNA$125 \mathrm{~b}$ expression in gastric adenocarcinoma and its effect on the proliferation of gastric cancer cells. Mol Med Rep 7: 229-232, 2013.

14. Zhi Q, Guo X, Guo L, et al: Oncogenic miR-544 is an important molecular target in gastric cancer. Anticancer Agents Med Chem 13: 270-275, 2013.

15. Guo JX, Tao QS, Lou PR, Chen XC, Chen J and Yuan GB: miR-181b as a potential molecular target for anticancer therapy of gastric neoplasms. Asian Pac J Cancer Prev 13: 2263-2267, 2012.

16. Tang H, Kong Y, Guo J, et al: Diallyl disulfide suppresses proliferation and induces apoptosis in human gastric cancer through Wnt-1 signaling pathway by up-regulation of miR-200b and miR-22. Cancer Lett 340: 72-81, 2013

17. Guo MM, Hu LH, Wang YQ, et al: miR-22 is down-regulated in gastric cancer, and its overexpression inhibits cell migration and invasion via targeting transcription factor Sp1. Med Oncol 30: $542,2013$.

18. Li B, Song Y, Liu TJ, et al: miRNA-22 suppresses colon cancer cell migration and invasion by inhibiting the expression of T-cel lymphoma invasion and metastasis 1 and matrix metalloproteinases 2 and 9. Oncol Rep 29: 1932-1938, 2013.

19. Ling B, Wang GX, Long G, Qiu JH and Hu ZL: Tumor suppressor miR-22 suppresses lung cancer cell progression through post-transcriptional regulation of ErbB3. J Cancer Res Clin Oncol 138: 1355-1361, 2012.

20. Xiong J, Yu D, Wei N, et al: An estrogen receptor alpha suppressor, microRNA-22, is downregulated in estrogen receptor alpha-positive human breast cancer cell lines and clinical samples. FEBS J 277: 1684-1694, 2010.
21. Wang W, Li F, Zhang Y, Tu Y, Yang Q and Gao X: Reduced expression of miR-22 in gastric cancer is related to clinicopathologic characteristics or patient prognosis. Diagn Pathol 8: 102, 2013.

22. Song SJ, Ito K, Ala U, et al: The oncogenic microRNA miR-22 targets the TET2 tumor suppressor to promote hematopoietic stem cell self-renewal and transformation. Cell Stem Cell 13: 87-101, 2013.

23. Kang DC, Su ZZ, Sarkar D, Emdad L, Volsky DJ and Fisher PB Cloning and characterization of HIV-1-inducible astrocyte elevated gene-1, AEG-1. Gene 353: 8-15, 2005.

24. Liu L, Wu J, Ying Z, et al: Astrocyte elevated gene-1 upregulates matrix metalloproteinase-9 and induces human glioma invasion. Cancer Res 70: 3750-3759, 2010

25. Ahn S, Hyeon J and Park CK: Metadherin is a prognostic predictor of hepatocellular carcinoma after curative hepatectomy. Gut Liver 7: 206-212, 2013

26. Wang N, Du X, Zang L, et al: Prognostic impact of Metadherin-SND1 interaction in colon cancer. Mol Biol Rep 39: 10497-10504, 2012.

27. Tokunaga E, Nakashima Y, Yamashita N, et al: Overexpression of metadherin/MTDH is associated with an aggressive phenotype and a poor prognosis in invasive breast cancer. Breast Cancer 21: 341-349, 2014.

28. Zhou J, Li J, Wang Z, Yin C and Zhang W: Metadherin is a novel prognostic marker for bladder cancer progression and overall patient survival. Asia Pac J Clin Oncol 8: e42-e48, 2012.

29. Meng X, Brachova P, Yang S, et al: Knockdown of MTDH sensitizes endometrial cancer cells to cell death induction by death receptor ligand TRAIL and HDAC inhibitor LBH589 co-treatment. PLoS One 6: e20920, 2011.

30. Ge X, Lv X, Feng L, et al: Metadherin contributes to the pathogenesis of diffuse large B-cell lymphoma. PLoS One 7: e39449, 2012.

31. Zhang J,Zhang Y, Liu S, et al: Metadherin confers chemoresistance of cervical cancer cells by inducing autophagy and activating ERK/NF-кB pathway. Tumour Biol 34: 2433-2440, 2013.

32. Hu G, Wei Y and Kang Y: The multifaceted role of MTDH/AEG-1 in cancer progression. Clin Cancer Res 15: 5615-5620, 2009.

33. Li X, Kong X, Huo Q, et al: Metadherin enhances the invasiveness of breast cancer cells by inducing epithelial to mesenchymal transition. Cancer Sci 102: 1151-1157, 2011.

34. Zhu K, Dai Z, Pan Q, et al: Metadherin promotes hepatocellular carcinoma metastasis through induction of epithelial-mesenchymal transition. Clin Cancer Res 17: 7294-7302, 2011. 